\title{
Modeling and Simulation of Segmented Primary Linear Induction Motor
}

\author{
Ying Jiang ${ }^{1,}$, Jingbo Shi ${ }^{2, b}$ \\ ${ }^{1}$ School of BEIHANG University, Beijing 100191, China; \\ 2 School of BEIHANG University, Beijing 100191, China. \\ ajy090672@163.com, ${ }^{b}$ Shijingbo@vip.sina.com
}

Keywords: linear induction motor, block feeding, long primary, simulation.

\begin{abstract}
The significance is increasing efficiency and saving energy for segmenting the long primary linear induction motor (LIM) into blocks. As the research object, a high order and non-linear model for single segment primary is built by using the finite element method (FEM), and the equations of inductance and induced electromotive force are established with the position of secondary as a parameter. Then, with the combination of the Ansoft circuit editor module and the Maxwell finite element module, the main circuit and the driving control circuit are designed. Finally, the system's dynamic characteristics are simulated and analyzed.by software Ansoft. The simulation results show that the proposed model can reflect the dynamic process of the system and the feasibility of the segmented feeding well.
\end{abstract}

\section{Introduction}

Linear motor can convert electrical energy into linear motion mechanical energy directly, which is widely used in industrial, transportation, national defense and other fields since the 1970s [1]. According to the relative length of the primary and secondary, it is divided into long primary LIM and short primary LIM [2, 3]. Long primary LIM make the secondary which is lightweight and without power supply as part of the movement, and this kind of structure is obvious superior to short primary in high speed and large thrust occasions [4]. But it is precisely make the leakage inductance large and the voltage utilization ratio low because of the long primary and short secondary structure, so it is significant to the segment the long primary into blocks and using block feeding methods for increasing efficiency $[5,6]$.At present, the domestic and foreign research is focus on the permanent magnet linear synchronous motor and linear induction motor [7].

That integrated modeling and simulation of the systems driven by multi-segment primary permanent linear synchronous motors is researched $[8,9]$. In this paper, a mathematical model for single segment primary is established which analogy to conventional rotatory motor. And then taking into account of the complexity of inductance and the flux linkage varying with secondary position change, this paper will linearize its virtual value reasonably to get the equation of inductance, induced electromotive force, electromagnetic force and motion equation. Finally, the system's dynamic characteristics are simulated and analyzed by using the finite element method.

\section{Modeling of Linear Induction Motor}

\section{Mathematical Model.}

It is necessary to build a system model for single segment primary, because each segmented primary is controlled independently to use block feeding method. For the segmented primary LIM, the secondary's position change can cause the coupling area increasing or decreasing between the primary and secondary in operating process. Therefore, the primary's magnetic circuit which is coupled with secondary and the electromagnetic parameters of the LIM will also be changed. So it is a crucial step to establish a non-linear and variable parameter model for single segment primary.

According to Ohm's law and the law of electromagnetic induction, the mathematical model of LIM is obtained as below.

Flux linkage equation: 


$$
\left[\begin{array}{l}
\psi_{A} \\
\psi_{B} \\
\psi_{C} \\
\psi_{a} \\
\psi_{b} \\
\psi_{c}
\end{array}\right]=\left[\begin{array}{llllll}
L_{A A} & L_{A B} & L_{A C} & L_{A a} & L_{A b} & L_{A c} \\
L_{B A} & L_{B B} & L_{B C} & L_{B a} & L_{B b} & L_{B c} \\
L_{C A} & L_{C B} & L_{C C} & L_{C a} & L_{C b} & L_{C c} \\
L_{a A} & L_{a B} & L_{a C} & L_{a a} & L_{a b} & L_{a c} \\
L_{b A} & L_{b B} & L_{b C} & L_{b a} & L_{b b} & L_{b c} \\
L_{c A} & L_{c B} & L_{c C} & L_{c a} & L_{c b} & L_{c c}
\end{array}\right]\left[\begin{array}{l}
i_{A} \\
i_{B} \\
i_{C} \\
i_{a} \\
i_{b} \\
i_{c}
\end{array}\right]
$$

Voltage equation:

$$
\left[\begin{array}{l}
u_{A} \\
u_{B} \\
u_{C} \\
u_{a} \\
u_{b} \\
u_{c}
\end{array}\right]=\left[\begin{array}{cccccc}
R_{s} & 0 & 0 & 0 & 0 & 0 \\
0 & R_{s} & 0 & 0 & 0 & 0 \\
0 & 0 & R_{s} & 0 & 0 & 0 \\
0 & 0 & 0 & R_{r} & 0 & 0 \\
0 & 0 & 0 & 0 & R_{r} & 0 \\
0 & 0 & 0 & 0 & 0 & R_{r}
\end{array}\right]\left[\begin{array}{l}
i_{A} \\
i_{B} \\
i_{C} \\
i_{a} \\
i_{b} \\
i_{c}
\end{array}\right]+p\left[\begin{array}{l}
\psi_{A} \\
\psi_{B} \\
\psi_{C} \\
\psi_{a} \\
\psi_{b} \\
\psi_{c}
\end{array}\right]
$$

Where $p$ refers to a differential operator for time.

Time-changes inductance is given by equation (3):

$$
p \boldsymbol{L}_{(\mathrm{x})}=\frac{d \boldsymbol{L}_{(\mathrm{x})}}{d t}=\frac{d \boldsymbol{L}_{(\mathrm{x})}}{d x} * \frac{d x}{d t}=v p_{x} \boldsymbol{L}_{(\mathrm{x})}
$$

Where $p_{x}$ refers to a differential operator for secondary displacement, $\boldsymbol{L}_{(x)}$ refers to inductance matrix, $v=\frac{d x}{d t}$ refers to the running speed of secondary.

\section{Electromotive Force (EMF) and Motion Equations.}

Based on equation (3), self-inductance, mutual inductance and excitation flux are changed with the secondary position, while this relationship is very difficult to use mathematical formula to express accurately. It is assumed that the inductance and excitation potential varies linearly with the secondary position, that is, the excitation flux linkage of the three-phase windings is a sine function about $\mathrm{x}$. The EMF can be obtained from the derivation for fundamental component of each phase winding flux through the finite element analysis.

$$
\begin{aligned}
& E_{A}=-\frac{d \psi_{A}(x)}{d t}=\psi_{m}(x) \bullet \frac{\pi}{\tau} \bullet \sin \left(\frac{\pi}{\tau} x\right) \\
& E_{B}=-\frac{d \psi_{B}(x)}{d t}=\psi_{m}\left(x-\frac{2 \tau}{3}\right) \bullet \frac{\pi}{\tau} \bullet \sin \frac{\pi}{\tau}\left(x-\frac{2 \tau}{3}\right) \\
& E_{C}=-\frac{d \psi_{C}(x)}{d t}=\psi_{m}\left(x-\frac{4 \tau}{3}\right) \bullet \frac{\pi}{\tau} \bullet \sin \frac{\pi}{\tau}\left(x-\frac{4 \tau}{3}\right)
\end{aligned}
$$

Based on equation (3), the EMF of primary section $\mathrm{k}$ will be deduced:

$$
\begin{aligned}
& E_{K A}=E_{A}\left[\varepsilon\left(x-x_{K A 1}\right)-\varepsilon\left(x-x_{K A 2}\right)\right] \\
& E_{K B}=E_{B}\left[\varepsilon\left(x-x_{K A 1}-\frac{2 \tau}{3}\right)-\varepsilon\left(x-x_{K A 2}-\frac{2 \tau}{3}\right)\right] \\
& E_{K C}=E_{C}\left[\varepsilon\left(x-x_{K A 1}-\frac{4 \tau}{3}\right)-\varepsilon\left(x-x_{K A 2}-\frac{4 \tau}{3}\right)\right]
\end{aligned}
$$

Where $\varepsilon(x)$ refers to unit step function, $x_{K A 1} 、 x_{K A 2}$ refers to the position of both ends for A phase winding of primary section $\mathrm{k}$.

The electromagnetic force of single segment primary:

$$
f_{e m}(x)=\frac{P_{e}}{v}
$$

Where $P_{e}$ refers to power of the winding 
The total electromagnetic force of LIM could be obtained from the following equation, where $\mathrm{k}$ refers to1, 2, 3, 4, 5:

$$
\sum f_{e m}(x)=\sum_{1}^{5} f_{e m}(x)_{k}
$$

Based on the analysis above, Motion equation could be obtained from the following equation, where $\mathrm{m}$ refers to the moving part mass, $f_{\text {load }}$ refers to load force, $f_{r}$ refers to friction or resistance.

\section{Running Process.}

The block feeding method is to shift the excited section at every block section which is coupling with secondary (Fig. 1).

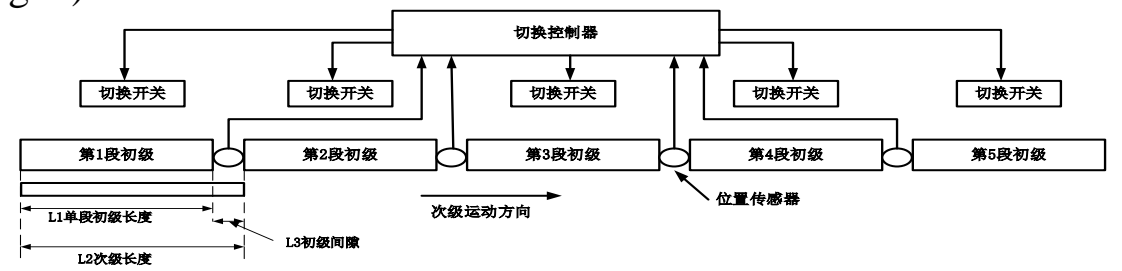

Fig. 1 Block feeding for long primary LIM

It is assumed that the secondary is in the initial position as shown in Figure 1 when $t=0$, where $x=0$. Firstly, the primary section 1 and 2 began to connect the power supply when switching controller received the signal from the position sensor. Then Electromagnetic force, produced with primary section 1 and secondary interaction, promoted the secondary to accelerate to couple with the primary section 2 and gradually withdraw from primary section 1 . Secondly, primary section 1 began to shift switch from on to off when the position sensor between the primary section 1 and 2 detected the secondary end leaving the primary section 1 . Similarly, primary section 3 began to shift switch from off to on when the position sensor between the primary section 2 and 3 detected the secondary end entering the primary section 3 . The rest could be deduced by analogy, so the order of each primary section's power supply was obtained as fellow: 1and2, 2and3, 3and4, 4and5. It can be guaranteed that the coupling area could be kept constant between primary with secondary, based on the equation: $L_{2}=L_{1}+L_{3}$, where $L_{1}$ refers to the length of single primary, $L_{2}$ refers to the length of secondary, $L_{3}$ refers to the distance between adjacent primary,

\section{Simulation Research}

\section{Driving Circuit.}

The realization of block feeding depends on positioning the secondary through position sensors so as to provide correct on-off control for three-phase voltage. Circuit editor in Ansoft could be used here for position variable $\mathrm{P}$, which can be used to get the displacement of secondary, and provide external power supply:

$$
\left\{\begin{array}{l}
u_{A}=220 \sqrt{2} \sin (2 \pi f t) \\
u_{B}=220 \sqrt{2} \sin (2 \pi f t-2 \pi / 3) \\
u_{C}=220 \sqrt{2} \sin (2 \pi f t+2 \pi / 3)
\end{array}\right.
$$

Based on equation (8), the main circuit and driving circuit model (Fig. 1) are built from element library in Maxwell Circuit Editor.

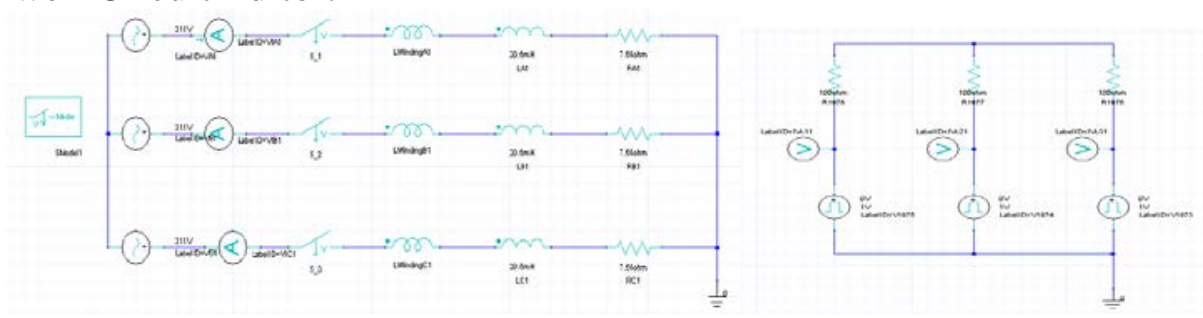

Fig. 2 Main circuit and driving circuit 


\section{Simulation Results.}

Table 1 displays the main parameters of the single long primary non - magnetic secondary linear induction motor used in this paper.

Table 1 The main parameters of long primary LIM

\begin{tabular}{|l|l|}
\hline Starting Thrust: $225 \mathrm{~N}$ & Synchronous Speed: $4.5 \mathrm{~m} / \mathrm{s}$ \\
\hline Voltage Rating: 380V & Supply Frequency: $50 \mathrm{~Hz}$ \\
\hline Phase Number: 3 & Pole-pairs Number(per primary): 3.5 \\
\hline Slots (per primary): 24 & Slots per pole per phase: 1 \\
\hline Tooth Width: 4mm & Tooth Pitch: $15 \mathrm{~mm}$ \\
\hline Pole Pitch(: 45mm & Mechanical Air Gap: $2 \mathrm{~mm}$ \\
\hline Winding Type: Double-layer with no compensation full-pitch winding, Y connection \\
\hline $\begin{array}{l}\text { Secondary Structure : steel copper compound, copper thickness: } 2 \mathrm{~mm} \text { 、 Steel plate } \\
\text { thickness :6mm, Length: } 370 \mathrm{~mm} \text {, width :110mm }\end{array}$ \\
\hline $\begin{array}{l}\text { Primary Structure : total length :1844mm, divided into five sections, each segment } \\
\text { length :364mm, each segment space: } 6 \mathrm{~mm} \text {, with half-filled slot at the end of primary windings. }\end{array}$
\end{tabular}

The simulation results show dynamic (including EMF and primary current ) characteristics from the first section to the fifth section primary as follows:

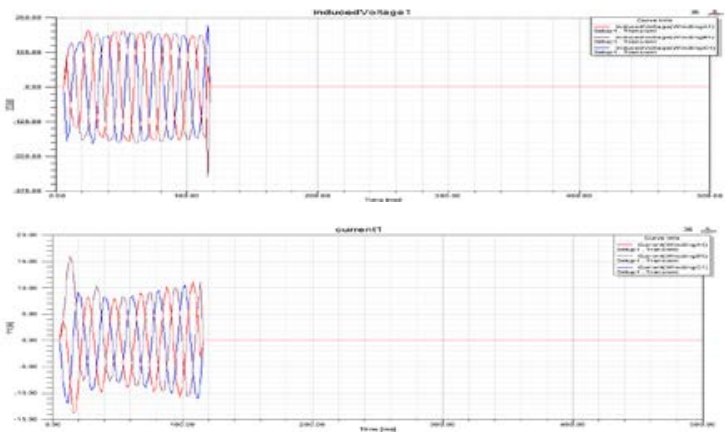

(a) The first section primary

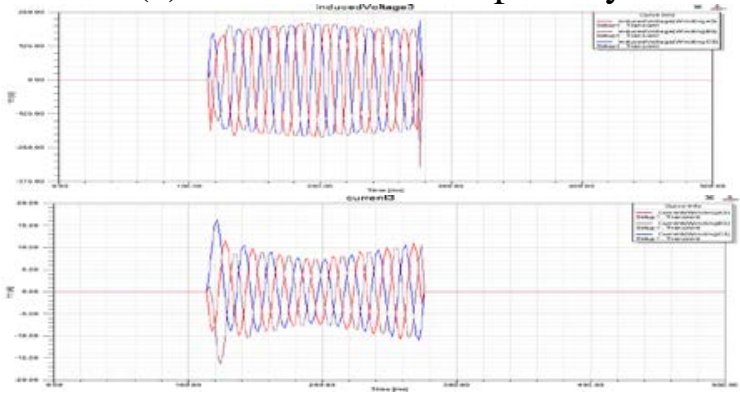

(c) The third section primary

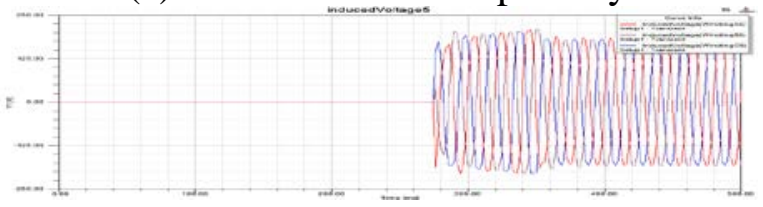

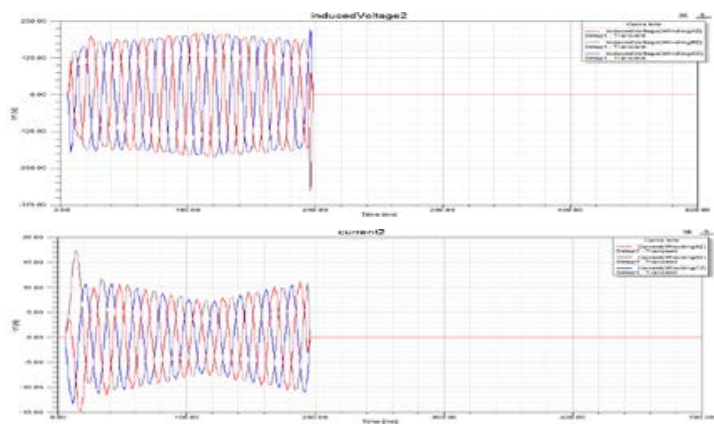

(b) The second section primary

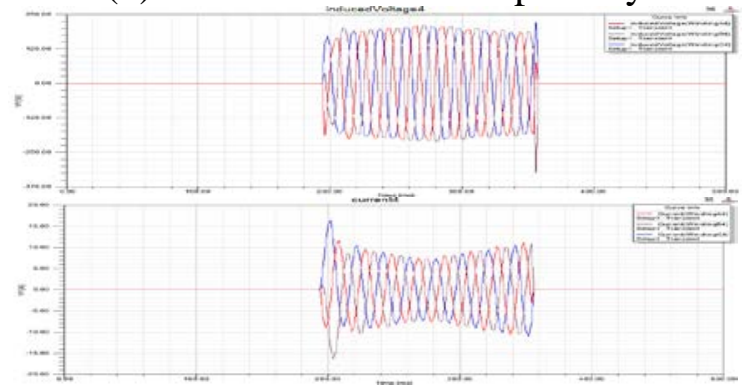

(d) The fourth section primary

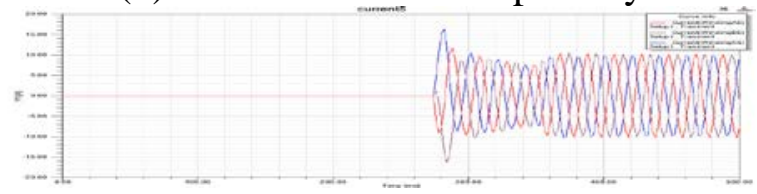

(e) The fifth section primary

Fig. 3 The dynamic characteristics for block feeding

Fig. 3 depict the primary current increasing immediately. Since each segmented primary is controlled independently to use block feeding method, the transient process of the air gap magnetic field is established in each independent model while multiple finite element analysis models need to be built respectively. In addition, ferrite core's disconnection which makes the magnetic circuit asymmetry lead to the emergence of unbalanced three-phase current caused by end effect.

In Fig. 3, the first section primary current keep increasing, and the current of the second, third and fourth have a process that increase first and then decrease, while the fifth maintain a downward trend, and it becomes locked-rotor current until the secondary reached the band boundary at $0.35 \mathrm{~s}$. 
Underpinning these poor performance is as above: (1) when the secondary enters into the primary, the inductance and the induced EMF increase with the coupling area between the secondary with the primary, and the armature current decreases according to Ohm's law. (2)When the secondary happens to be fully covered in a certain primary, the primary inductance, the induced EMF and current should be kept constant. (3)When the secondary exit from the primary, the result is just the opposite to the situation 1, that is, the inductance and the induced EMF decrease while the armature current increases.

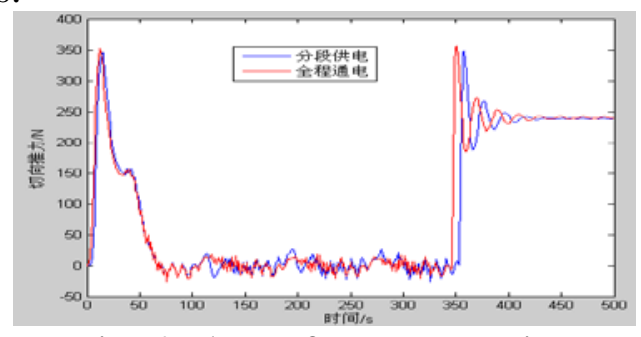

Fig. 4 Thrust force comparing

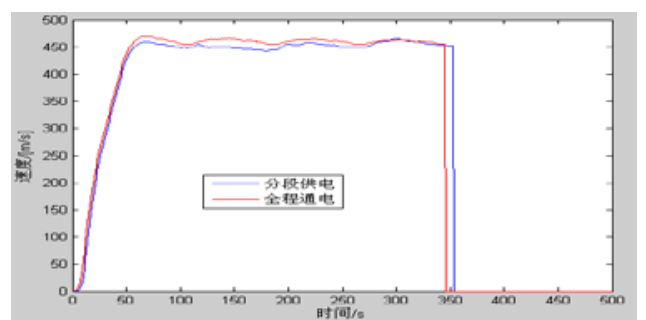

Fig. 5 Secondary speed comparing

The ordinary feeding method is to supply power in whole system. On the other hand, the proposed method is to shift the excited section at every block section. Respectively, Fig. 4 depict the thrust force comparing while Fig. 5 shows the secondary speed comparing. Within the limit of error, the simulation curves starting thrust basically coincide, and it's also suitable for secondary speed. It can be seen that block feeding supply can reach the target by use the ordinary feeding method. The simulation results also prove the rationality of the block feeding theory.

\section{Summary}

Selecting the segmented long primary linear induction motor as the research object, this paper established a high order, nonlinear, strong coupling mathematical model of the single segment primary based on a reasonable assumption. Equations of inductance, induction electric potential and motion, changing with the secondary displacement, were acquired after a reasonable calculation. Then the position signal P in Maxwell circuit Editor was used to position the secondary displacement to achieve an accurate control of the LIM power supply. The simulation results show that, as the secondary gets into a certain primary, induction electric potential and inductance of the primary will increase with the coupling area between them, which will lead to the decreasing of the armature current. A totally opposite result will acquired as the secondary exits one primary. Finally, we compared the thrust and speed of block feeding supply to those of power supply in whole system, and it shows that block feeding supply can achieve the same effect of power supply in whole system, while reducing the demand for power supply as the same time. Therefore the conclusions and simulation obtained in this paper will provide a reference for the segmentation design and control of the long primary linear induction motor.

\section{References}

[1]. Ye Yunyue. Principle and application of linear motor[M]. BeiJing:Machinery Industry Press,2000, 19-24(in Chinese).

[2]. Nasar S A, Boldea I. Linear motion electric machine[M]. New York:Wiley, 1976, 30-50.

[3]. Ion Boldea, Nasar S A. Linear motion electromagnetic devices[M]. New York:Taylor\&Francis, 2001, 15-20.

[4]. Lu Junyong, Ma Weiming. Xu Jin. Modeling and simulation of high speed long primary double-sided linear induction motor[J]. Proceedings of the CSEE, 2008, 28(27): 89-94(in Chinese).

[5]. Cui Xiaopeng, Wang Gongbao, Ma Weiming. Research on fault diagnosis of segment-powered linear induction motor[J]. Electric Machines and Control, 2013, 17(8): 9-14(in Chinese). 
[6]. Onuki T, Kamiya Y, Kurimoto Y, et al. A novel block feeding method in the single-sided LIM with a short secondary member[J]. IEEE Transaction on Energy Conversion, 1999, 14(4): 1323-1328.

[7]. Zhang Mingyuan, Ma Weiming. He Na, et al. Application of block feeding methods in long primary linear motors[J]. Chinese Electrical Engineering Science, 2013, 33(27): 9-14(in Chinese).

[8]. Shangguan Xuanfeng, Li Qingfu, Yuan Shiying, et al. Analysis on running process of permanent linear synchronous motors with discontinuous stators[J]. Journal of Xi'an Jiaotong University, 2004, 38(12): 1292-1295(in Chinese).

[9]. Shangguan Xuanfeng, Li Qingfu, Yuan Shiying, et al. Integrated modeling and simulation of the system driven by multi-segment primary permanent linear synchronous motors[J]. Transactions of China Electrotechnical Society, 2006, 21(3): 52-57(in Chinese). 